\title{
Distribution of values of Euler's function over integers free of large prime factors
}

by

\author{
A. Smati (Limoges) and J. Wu (Nancy)
}

1. Introduction. Let $\varphi(n)$ be Euler's function and let $A(x):=\sum_{\varphi(n) \leq x} 1$. Erdős and Turán [5] established the asymptotic relation

$$
A(x) \sim A x \quad(x \rightarrow \infty)
$$

with

$$
A:=\prod_{p}\left(1+\frac{1}{p(p-1)}\right)=1.9435 \ldots,
$$

where $p$ ranges over all prime numbers. Using analytic methods, Bateman [2] proved that for any $\varepsilon>0$ the error term of (1.1) is

$$
\ll_{\varepsilon} x \exp \left\{-(1 / \sqrt{2}-\varepsilon) \sqrt{\log x \log _{2} x}\right\} .
$$

In 1989, Balazard and the first author [1] gave an elementary proof of (1.2). As it seldom occurs that an elementary result reaches the degree of accuracy of the best known analytic method, we may say that this elementary method is effective. Very recently we succeeded, using the same argument, in generalizing Bateman's theorem to the case of algebraic number fields [13].

In this paper, we shall further develop this method to investigate distribution of values of Euler's function over integers free of large prime factors. Let $P(n)$ be the largest prime factor of the integer $n>1$, with the convention that $P(1)=1$. Defining, for $x \geq y \geq 2$,

$$
A(x, y):=\sum_{\varphi(n) \leq x, P(n) \leq y} 1,
$$

we are interested in the asymptotic behaviour of this function. In view of (1.1), we could expect that $A(x, y) \sim A \Psi(x, y)$ holds under suitable conditions on $x$ and $y$, where

$$
\Psi(x, y):=\sum_{n \leq x, P(n) \leq y} 1
$$


denotes the number of positive integers $\leq x$ and free of prime factors $>y$. The latter has been extensively studied by various authors. An excellent survey, including an exhaustive bibliography, has been written by Hildebrand and Tenenbaum [9]. For $x, y \geq 2$, we shall systematically use the notation $u:=\log x / \log y$. This quantity will play a pivotal role in the study of the asymptotic behaviour of $A(x, y)$. Denote by $\log _{k}$ the $k$-fold iterated logarithm.

Our principal result is as follows.

THEOREM 1. For any $\varepsilon>0$, the asymptotic relation

$$
A(x, y)=\Psi(x, y)\left\{A+O_{\varepsilon}\left(\frac{\log u+\log _{2} y \log _{3} y}{\log y}\right)\right\}
$$

holds uniformly in the range

$$
y \geq 2, \quad 1 \leq u \leq \exp \left\{\varepsilon^{-1} \sqrt{\log y}\right\} .
$$

From Theorem 1, we immediately deduce the following result.

Corollary 1. In the range (1.4), we have $A(x, y) \sim A \Psi(x, y)$ if $y \rightarrow \infty$.

Let $\varrho(u)$ be Dickman's function, which is defined as the unique solution, which is continuous at $u=1$ and differentiable for $u>1$, of the differencedifferential equation

$$
u \varrho^{\prime}(u)=-\varrho(u-1) \quad(u>1)
$$

with initial condition $\varrho(u)=1(0 \leq u \leq 1)$. The following formula, due to de Bruijn [3], describes the asymptotic behaviour of $\log \varrho(u)$ :

$$
\varrho(u)=\exp \left\{-u\left(\log u+\log _{2}(u+2)-1+O\left(\frac{\log _{2}(u+2)}{\log (u+2)}\right)\right)\right\}
$$

for $u \geq 1$. Combining Theorem 1 with a well-known theorem of Hildebrand (cf. Lemma 2.3 below), we immediately get the following statement.

COROLlary 2. For any $\varepsilon>0$, the asymptotic formula

$$
A(x, y)=x \varrho(u)\left\{A+O_{\varepsilon}\left(\frac{\log u+\log _{2} y \log _{3} y}{\log y}\right)\right\}
$$

holds uniformly in the range (1.4). by

For proving Theorem 1, we approximate, as in [1], Euler's function $\varphi(n)$

$$
\varphi(n, z):=n \prod_{p \mid n, p \leq z}\left(1-p^{-1}\right) \quad(z \geq 2)
$$


and we consider the sum

$$
A(x, y, z):=\sum_{\varphi(n, z) \leq x, P(n) \leq y} 1 \quad(x \geq y \geq z \geq 2) .
$$

Let $\omega(n)$ be the number of distinct prime factors of the integer $n$. We have $\omega(n) \leq \log n / \log 2$. In addition, for $\varphi(n) \leq x$ we find that $n \ll x \log _{2} x$ and $\log n \leq\{1+o(1)\} \log x(x \rightarrow \infty)$. Hence we can deduce that

$$
\begin{aligned}
\varphi(n, z) & \geq \varphi(n)=\varphi(n, z) \prod_{p \mid n, p>z}\left(1-p^{-1}\right) \geq \varphi(n, z)\left(1-z^{-1}\right)^{\omega(n)} \\
& \geq \varphi(n, z)(1-\log n /(z \log 2)) \geq \varphi(n, z)(1-\omega(n) / z) \\
& \geq \varphi(n, z)(1-3 \log x / z),
\end{aligned}
$$

which implies the following simple bounds:

$$
A(x, y, z) \leq A(x, y) \leq A\left(\frac{x}{1-3 \log x / z}, y, z\right) .
$$

In order to evaluate $A(x, y, z)$, we observe that

$$
A(x, y, z)=\sum_{\varphi(n) \leq x / z, P(n) \leq z} \Theta\left(\frac{x}{\varphi(n)}, y, z\right)+A(x, z)
$$

where

$$
\Theta(x, y, z):=|\{n \leq x: p \mid n \Rightarrow z<p \leq y\}| .
$$

With the aid of a theorem of Saias (see below Lemma 2.1), we can derive a precise estimate for $A(x, y, z)$ (see Lemma 3.6 below). Finally, Theorem 1 follows from a suitable choice of $z$.

As for applications of Theorem 1, it seems interesting to consider the sums

$$
\sum_{\varphi(n) \leq x} 1 / P(n), \quad \sum_{\varphi(n) \leq x} \log P(n),
$$

which can be regarded as analogues of $\sum_{n \leq x} 1 / P(n)$ and $\sum_{n \leq x} \log P(n)$. In 1986, Erdős, Ivić and Pomerance [6] obtained

$$
\sum_{n \leq x} \frac{1}{P(n)}=x \delta(x)\left\{1+O\left(\left(\frac{\log _{2} x}{\log x}\right)^{1 / 2}\right)\right\},
$$

where $\delta(x):=\int_{2}^{x} \varrho(\log x / \log t) t^{-2} d t$. Using (1.5), they proved that

$$
\begin{aligned}
\delta(x)=\exp \left\{-\sqrt{2 \log x \log _{2} x}(1+\right. & \frac{\log _{3} x-2-\log 2}{2 \log _{2} x} \\
& \left.\left.-\left\{\frac{1}{8}+o(1)\right\}\left(\frac{\log _{3} x}{\log _{2} x}\right)^{2}\right)\right\} .
\end{aligned}
$$


In 1990, Tenenbaum ([14], Exercise III.5.3) showed that, for any $\varepsilon>0$,

$$
\sum_{n \leq x} \log P(n)=\lambda x \log x-\lambda(1-\gamma) x+O_{\varepsilon}\left(x \exp \left\{-(\log x)^{3 / 8-\varepsilon}\right\}\right)
$$

with $\lambda:=1-\int_{1}^{\infty} \varrho(t) t^{-2} d t=0.6243 \ldots$

We have the following results.

Theorem 2. For $x \geq 2$,

$$
\sum_{\varphi(n) \leq x} \frac{1}{P(n)}=x \delta(x)\left\{A+O\left(\left(\frac{\log _{2} x}{\log x}\right)^{1 / 2}\right)\right\} .
$$

TheOREM 3. For $x \geq 2$,

$$
\sum_{\varphi(n) \leq x} \log P(n)=A \lambda x \log x+O\left(x \log _{2} x \log _{3} x\right) .
$$

Acknowledgement. The authors would like to thank A. Ivić who suggested them to study this problem.

2. Notations and auxiliary lemmas. For $u>1$, we define $\xi=\xi(u)$ as the unique positive solution of the equation $e^{\xi}=1+u \xi$, with the convention that $\xi(u)=0(0<u \leq 1)$. For $x, y \geq 2$, let $\alpha=\alpha(x, y)$ be the unique solution of the equation

$$
\log x=\sum_{p \leq y} \frac{\log p}{p^{\sigma}-1} .
$$

For $x, y, z \geq 2$ and $d \geq 1$, we set

$$
\begin{aligned}
u & :=\frac{\log x}{\log y}, \quad r:=\frac{\log z}{\log y}, \quad \beta=\beta(x, y):=1-\frac{\xi(u)}{\log y}, \\
u_{d} & :=\frac{\log (x / d)}{\log y}, \quad \alpha_{d}:=\alpha\left(\frac{x}{d}, y\right), \quad \beta_{d}:=\beta\left(\frac{x}{d}, y\right) .
\end{aligned}
$$

Denote by $\varepsilon$ a sufficiently small positive number and put $L_{\varepsilon}(y):=$ $\exp \left\{(\log y)^{3 / 5-\varepsilon}\right\}$. We use $c_{1}, c_{2}, \ldots$ to denote positive absolute constants.

Next we shall cite some known results, which will be needed in the proofs of Theorems 1 and 2. The first lemma is an immediate consequence of Théorème 7 of Saias [12], which precisely describes the asymptotic behaviour of $\Theta(x, y, z)$.

LEMMA 2.1. Under the condition (1.4), the asymptotic formula

$$
\Theta(x, y, z)=\Psi(x, y) \prod_{p \leq z}\left(1-p^{-\alpha}\right)\left\{1+O_{\varepsilon}\left(r e^{-(1-2 r) \log u}+e^{-1 / r}\right)\right\}
$$

holds uniformly for $2 u \leq z \leq y^{1 / 2}$. 
The following result is due to de Bruijn [4] and Hildebrand [7]. Here we take this in the form of [9].

Lemma 2.2 ([9], Theorem 1.2). For any $\varepsilon>0$, the asymptotic formula

$$
\log (\Psi(x, y) / x)=\left\{1+O_{\varepsilon}\left(1 / L_{\varepsilon}(u)\right)\right\} \log \varrho(u)
$$

holds uniformly in the range

$$
x \geq 2, \quad(\log x)^{1+\varepsilon} \leq y \leq x .
$$

Moreover, the lower bound in (2.1) holds uniformly for all $x \geq y \geq 2$.

The third lemma is an important result of Hildebrand [7] about $\Psi(x, y)$.

Lemma 2.3 ([7], Theorem 1). For any $\varepsilon>0$, the asymptotic formula

$$
\Psi(x, y)=x \varrho(u)\left\{1+O_{\varepsilon}\left(\frac{\log (u+1)}{\log y}\right)\right\}
$$

holds uniformly in the range

$$
y \geq 2, \quad 1 \leq u \leq \exp \left\{(\log y)^{3 / 5-\varepsilon}\right\} .
$$

The next three lemmas, due to Ivić-Tenenbaum [11] and HildebrandTenenbaum [8], describe the local behaviour of $\Psi(x, y)$.

Lemma 2.4 ([11], Lemma 2). Under the condition $\left(\mathrm{R}_{\varepsilon}\right)$, the asymptotic formula

$$
\Psi(x / d, y)=\Psi(x, y) d^{-\beta}\left\{1+O_{\varepsilon}\left(\frac{\log _{2} y}{\log y}+\frac{\log d}{\log x}\right)\right\}
$$

holds uniformly for $1 \leq d \leq y$.

Lemma 2.5 ([11], Lemma 3). Under the condition $\left(\mathrm{R}_{\varepsilon}\right)$, the asymptotic formula

$$
\Psi(x / d, y) \ll_{\varepsilon} \Psi(x, y) d^{-\beta+c_{1} / \log y}
$$

holds uniformly for $1 \leq d \leq x$, where $c_{1}$ is a positive absolute constant.

LEMma 2.6 ([8], Theorem 3). We have, uniformly for $x \geq y \geq 2$ and $1 \leq c \leq y$,

$$
\Psi(c x, y)=\Psi(x, y) c^{\alpha(x, y)}\left\{1+O\left(\frac{1}{u}+\frac{\log y}{y}\right)\right\} .
$$

From (2.10) of [8], we immediately see the following statement.

Lemma 2.7. Under the condition $\left(\mathrm{R}_{\varepsilon}\right)$, the asymptotic formula

$$
\Psi(x+x / z, y)=\Psi(x, y)\left\{1+O_{\varepsilon}(1 / z)\right\}
$$

holds uniformly for $1 \leq z \leq y$.

The last lemma, due to Ivić [10], is useful to prove Theorem 2. 
Lemma 2.8 ([10], (4.1) and (4.3)). For any fixed $\theta>0$, we have

$\sum_{n \leq x} \frac{1}{P(n)}=1+\sum_{p \leq x} \frac{1}{p} \Psi\left(\frac{x}{p}, p\right)=\left\{1+O_{\theta}\left(\frac{1}{(\log x)^{\theta}}\right)\right\} \sum_{L_{-} \leq p \leq L_{+}} \frac{1}{p} \Psi\left(\frac{x}{p}, p\right)$, where $L_{ \pm}:=\exp \left\{\sqrt{(1 / 2) \log x \log _{2} x}\left(1 \pm 2 \log _{3} x / \log _{2} x\right)\right\}$.

3. Proof of Theorem 1. Before proving Theorem 1, we first prove preliminary lemmas.

Lemma 3.1. Defining $A(\sigma, z):=\prod_{p \leq z}\left(1+(p-1)^{-\sigma}-p^{-\sigma}\right)$, we have, uniformly for $z \geq 2$ and $0<\sigma_{0} \leq \sigma \leq 1$,

$$
A(\sigma, z)=A+O_{\sigma_{0}}\left(|1-\sigma|+z^{-\sigma}\right) .
$$

Proof. For any $\sigma>0$ we have

$$
0 \leq(p-1)^{-\sigma}-p^{-\sigma}=\sigma \int_{p-1}^{p} t^{-\sigma-1} d t \leq \sigma(p-1)^{-\sigma-1} .
$$

Upon putting $A(\sigma):=A(\sigma, \infty)$, this inequality allows us to write, for $z \geq 2$ and $0<\sigma_{0} \leq \sigma \leq 1$,

$$
\begin{aligned}
A(\sigma, z) & =A(\sigma) \exp \left\{O\left(\sigma \sum_{p>z}(p-1)^{-\sigma-1}\right)\right\} \\
& =A(\sigma) \exp \left\{O\left(z^{-\sigma}\right)\right\}=A(\sigma)+O_{\sigma_{0}}\left(z^{-\sigma}\right) .
\end{aligned}
$$

In addition, (3.1) implies that

$$
\begin{aligned}
\frac{A^{\prime}}{A}(\sigma) & =-\sum_{p} \frac{\left((p-1)^{-\sigma}-p^{-\sigma}\right) \log p+(p-1)^{-\sigma} \log (1-1 / p)}{1+(p-1)^{-\sigma}-p^{-\sigma}} \\
& \ll \sum_{p} p^{-1-\sigma} \log p \ll_{\sigma_{0}} 1 .
\end{aligned}
$$

Hence for $0<\sigma_{0} \leq \sigma \leq 1$ we deduce that

$$
A(\sigma)=A \exp \left\{-\int_{\sigma}^{1} \frac{A^{\prime}}{A}(t) d t\right\}=A+O_{\sigma_{0}}(|1-\sigma|) .
$$

Now the required result follows from (3.2) and (3.3).

LEMMA 3.2. Under the condition $\left(\mathrm{R}_{\varepsilon}\right)$, the asymptotic formula

$$
\prod_{p \leq z}\left(1-p^{-\alpha}\right)=\left\{1+O_{\varepsilon}\left(\frac{\log (u+1)}{\log y}\right)\right\} \prod_{p \leq z}\left(1-p^{-\beta}\right)
$$

holds uniformly for $2 \leq z \leq \min \left\{y, \exp \left\{(\log y)^{8 / 5-\varepsilon} / \xi(u)\right\}\right\}$. 
Proof. First we observe that

$$
\prod_{p \leq z} \frac{1-p^{-\alpha}}{1-p^{-\beta}}=\exp \left\{\int_{\beta}^{\alpha} \sum_{p \leq z} \frac{\log p}{p^{\sigma}-1} d \sigma\right\} .
$$

Since $\alpha, \beta \gg_{\varepsilon} 1$ in the range $\left(\mathrm{R}_{\varepsilon}\right)$ (cf. (7.8) of [8]), Lemma 13 of [8] allows us to write

$$
\int_{\beta}^{\alpha} \sum_{p \leq z} \frac{\log p}{p^{\sigma}-1} d \sigma=\left\{1+O_{\varepsilon}\left(\frac{1}{L_{\varepsilon}(z)}\right)\right\} \int_{\beta}^{\alpha} \int_{1}^{z} t^{-\sigma} d t d \sigma+O_{\varepsilon}(|\alpha-\beta|) .
$$

Using (7.8) of [8] and noticing $2 \leq z \leq y$, we can find that

$$
\begin{aligned}
\left|\int_{\beta}^{\alpha} \int_{1}^{\alpha} t^{-\sigma} d t d \sigma\right| & =\left|\int_{\beta}^{\alpha} \frac{z^{1-\sigma}-1}{1-\sigma} d \sigma\right| \leq\left|\int_{\beta}^{\alpha} z^{1-\sigma} \log z d \sigma\right|=\left|z^{1-\beta}-z^{1-\alpha}\right| \\
& \leq z^{\max \{1-\beta, 1-\alpha\}}(\log z)|\alpha-\beta| \\
& \ll z^{\xi(u) / \log y}(\log z)\left(\frac{1}{L_{\varepsilon / 2}(y)}+\frac{1}{u(\log y)^{2}}\right) .
\end{aligned}
$$

Under the hypothesis $2 \leq z \leq \min \left\{y, \exp \left\{(\log y)^{8 / 5-\varepsilon} / \xi(u)\right\}\right\}$, it is easy to verify

$$
z^{\xi(u) / \log y}(\log z)\left(\frac{1}{L_{\varepsilon / 2}(y)}+\frac{1}{u(\log y)^{2}}\right) \ll \frac{\log (u+1)}{\log y} .
$$

This proves that

$$
\int_{\beta}^{\alpha} \sum_{p \leq z} \frac{\log p}{p^{\sigma}-1} d \sigma \ll_{\varepsilon} \frac{\log (u+1)}{\log y} .
$$

In view of (3.4), this is equivalent to the desired result.

LEMMA 3.3. In the range

$$
x \geq 2, \quad(\log x)^{1+\varepsilon} \leq y \leq \sqrt{x},
$$

we have

$$
\prod_{p \leq z}\left(1-p^{-\beta_{d}}\right)=\left\{1+O_{\varepsilon}\left(\frac{\log z}{\log x}\right)\right\} \prod_{p \leq z}\left(1-p^{-\beta}\right)
$$

uniformly for $1 \leq d \leq y$ and $2 \leq z \leq \exp \left\{\varepsilon^{-3}(\log y) / \xi(u)\right\}$. Further, under the condition $\left(\mathrm{R}_{\varepsilon}\right)$, we have

$$
\prod_{p \leq z}\left(1-p^{-\beta_{d}}\right) \ll_{\varepsilon} \prod_{p \leq z}\left(1-p^{-\beta}\right)
$$

uniformly for $1 \leq d \leq x$ and $2 \leq z \leq \exp \left\{\varepsilon^{-3}(\log y) / \xi(u)\right\}$. 
Proof. In the range $\left(\mathrm{R}_{\varepsilon}^{\prime}\right)$ and for $1 \leq d \leq y$, we have $2 \leq u \leq y^{1-\varepsilon / 2}$ and $1 \leq u / 2 \leq u_{d} \leq u$. Thus it follows (cf. Lemma 2.2 of [9]) that

$$
\begin{gathered}
\beta_{d}-\beta=\frac{\xi(u)-\xi\left(u_{d}\right)}{\log y} \ll \frac{u-u_{d}}{u \log y} \ll \frac{\log d}{u(\log y)^{2}} \ll \frac{1}{\log x}, \\
\int_{\beta}^{\beta_{d}} \int_{1}^{z} t^{-\sigma} d t d \sigma \leq z^{1-\beta}(\log z)\left|\beta_{d}-\beta\right| \ll \frac{z^{\xi(u) / \log y} \log z}{\log x} \ll \varepsilon \frac{\log z}{\log x},
\end{gathered}
$$

since $1 \leq d \leq y$ and $2 \leq z \leq y$. Now these estimates imply, via (3.4) and (3.5), the assertion (3.6).

In the range $\left(\mathrm{R}_{\varepsilon}\right)$ and for $1 \leq d \leq x$, we have trivially

$$
\begin{gathered}
\beta_{d}-\beta=\frac{\xi(u)-\xi\left(u_{d}\right)}{\log y} \leq \frac{\xi(u)}{\log y} \ll_{\varepsilon} 1, \\
\int_{\beta}^{\beta_{d} z} \int_{1}^{z} t^{-\sigma} d t d \sigma \leq z^{1-\beta}(\log z)\left|\beta_{d}-\beta\right| \leq z^{\xi(u) / \log y} \frac{\xi(u) \log z}{\log y} \ll_{\varepsilon} 1 .
\end{gathered}
$$

Combining these estimates with (3.4) and (3.5) leads to the inequality (3.7).

LEMma 3.4. Under the condition $\left(\mathrm{R}_{\varepsilon}\right)$, we have

$$
\sum_{\varphi(n)>y, P(n) \leq z} \varphi(n)^{-\beta+c_{1} / \log y} \prod_{p \leq z}\left(1-p^{-\beta}\right) \ll_{\varepsilon} e^{-1 / r}
$$

uniformly for

$$
2 \leq z \leq \min \left\{\exp \left\{(\log y)^{1 / 2+\varepsilon / 5}\right\}, \exp \left\{\log y \log _{2} y /(2+\varepsilon) \xi(u)\right\}\right\} .
$$

Proof. Let $S_{0}$ be the quantity to be estimated. By Rankin's method, for any $\eta \in(0,1 / 10]$ we can write

$$
S_{0} \leq y^{-\eta} \sum_{P(n) \leq z} \varphi(n)^{-\beta+\eta+c_{1} / \log y} \prod_{p \leq z}\left(1-p^{-\beta}\right) .
$$

By noticing that, for any $\sigma>0$ and $z \geq 2$,

$$
\sum_{P(n) \leq z} \varphi(n)^{-\sigma} \prod_{p \leq z}\left(1-p^{-\sigma}\right)=A(\sigma, z),
$$

Lemma 3.1 and (3.4) and (3.5) yield that

$$
\begin{aligned}
S_{0} & \ll y^{-\eta} \prod_{p \leq z} \frac{1-p^{-\beta}}{1-p^{-\beta+\eta+c_{1} / \log y}} \\
& \ll y^{-\eta} \exp \left\{c_{2} \int_{\beta-\eta-c_{1} / \log y}^{\beta} \int_{1}^{z} t^{-\sigma} d t d \sigma+c_{3} \eta\right\} \\
& \ll y^{-\eta} \exp \left\{c_{4} z^{1-\beta+\eta+c_{1} / \log y}(\log z) \eta\right\}
\end{aligned}
$$




$$
\ll y^{-\eta} \exp \left\{c_{4} z^{\xi(u) / \log y+\eta+c_{1} / \log y}(\log z) \eta\right\} .
$$

Now the required result follows on taking $\eta=2 / \log z$.

Lemma 3.5. Under the condition $\left(\mathrm{R}_{\varepsilon}\right)$, we have

$$
\sum_{\varphi(n) \leq y, P(n) \leq z} \varphi(n)^{-\beta} \log \varphi(n) \prod_{p \leq z}\left(1-p^{-\beta}\right) \ll_{\varepsilon} \log z \log _{2} z
$$

uniformly for $2 \leq z \leq \exp \left\{\varepsilon^{-3}(\log y) / \xi(u)\right\}$.

Proof. Let $Y \geq 2$ be a parameter to be chosen later. We define

$$
\begin{aligned}
& S_{1}:=\sum_{\varphi(n) \leq Y, P(n) \leq z} \varphi(n)^{-\beta} \log \varphi(n) \prod_{p \leq z}\left(1-p^{-\beta}\right), \\
& S_{2}:=\sum_{Y<\varphi(n) \leq y, P(n) \leq z} \varphi(n)^{-\beta} \log \varphi(n) \prod_{p \leq z}\left(1-p^{-\beta}\right) .
\end{aligned}
$$

The relation (3.8) and Lemma 3.1 imply immediately

$$
S_{1} \leq A(\beta, z) \log Y \ll \log Y .
$$

When $Y<\varphi(n) \leq y$, we easily see that $\log \varphi(n) \leq \varphi(n)^{\delta}$ with $\delta:=$ $\log _{2} Y / \log Y$, which implies

$$
S_{2} \leq \sum_{\varphi(n)>Y, P(n) \leq z} \varphi(n)^{-\beta+\delta} \prod_{p \leq z}\left(1-p^{-\beta}\right) .
$$

As in the proof of Lemma 3.4, for any $\eta \in(0,1 / 10]$ we write

$$
S_{2} \leq Y^{-\eta} \prod_{p \leq z} \frac{1-p^{-\beta}}{1-p^{-\beta+\delta+\eta}} \ll Y^{-\eta} \exp \left\{c_{5} z^{1-\beta+\eta+\delta}(\log z)(\delta+\eta)\right\} .
$$

Taking $\eta=1 / \log z$ leads to the estimate

$$
S_{2} \ll \exp \left\{-\log Y / \log z+c_{5} e^{1 / \varepsilon^{3}+1} z^{\delta}(1+\delta \log z)\right\} .
$$

Combining (3.9) and (3.10) and taking $Y=\exp \left\{\log z \log _{2} z\right\}$, we obtain the desired result.

LEMMA 3.6. In the range

$$
y \geq 2, \quad 2 \leq u \leq \exp \left\{\varepsilon^{-1} \sqrt{\log y}\right\},
$$

we have

uniformly for

$$
A(x, y, z)=\Psi(x, y)\left\{A+O_{\varepsilon}\left(R_{1}\right)\right\}
$$

$$
(u \log y)^{1+\varepsilon} \leq z \leq \min \left\{\exp \left\{(\log y)^{1 / 2+\varepsilon / 5}\right\}, \exp \left\{\varepsilon^{-3}(\log y) / \xi(u)\right\}\right\},
$$

where

$R_{1}:=\frac{\log u}{\log y}+\frac{\log _{2} y}{\log y}+\frac{\log z \log _{2} z}{\log x}+r e^{-(1-2 r) \log u}+e^{-1 / r}+\frac{e^{r \xi(u)}}{z}+r^{5} \log _{2} x$. 
Proof. We start by the expression (1.7). To evaluate the sum on the right-hand side of (1.7), we divide the range of summation into two parts: $\varphi(n) \leq y$ and $y<\varphi(n) \leq x / z$. The corresponding contributions $S_{3}$ and $S_{4}$ can be estimated as follows.

From Lemma 2.1, we deduce that

$$
\begin{aligned}
S_{3}=\sum_{\varphi(n) \leq y, P(n) \leq z} \Psi\left(\frac{x}{\varphi(n)},\right. & y) \prod_{p \leq z}\left(1-p^{-\alpha_{\varphi(n)}}\right) \\
& \times\left\{1+O\left(r e^{-(1-2 r) \log u}+e^{-1 / r}\right)\right\} .
\end{aligned}
$$

Lemmas 3.2 and 3.3 imply

$$
\prod_{p \leq z}\left(1-p^{-\alpha_{\varphi(n)}}\right)=\left\{1+O\left(\frac{\log u}{\log y}+\frac{\log z}{\log x}\right)\right\} \prod_{p \leq z}\left(1-p^{-\beta}\right) .
$$

Inserting into (3.11) and applying Lemma 2.4 to evaluate $\Psi(x / \varphi(n), y)$, we obtain

$$
\begin{aligned}
S_{3} & =\Psi(x, y) \sum_{\varphi(n) \leq y, P(n) \leq z} \varphi(n)^{-\beta} \prod_{p \leq z}\left(1-p^{-\beta}\right) \\
& \times\left\{1+O\left(\frac{\log u}{\log y}+\frac{\log _{2} y}{\log y}+\frac{\log z}{\log x}+\frac{\log \varphi(n)}{\log x}+r e^{-(1-2 r) \log u}+e^{-1 / r}\right)\right\} .
\end{aligned}
$$

In addition, in view of (3.8), Lemmas 3.1 and 3.4 yield that

$$
\sum_{\varphi(n) \leq y, P(n) \leq z} \varphi(n)^{-\beta} \prod_{p \leq z}\left(1-p^{-\beta}\right)=A+O\left(\frac{\log u}{\log y}+\frac{e^{r \xi(u)}}{z}+e^{-1 / r}\right) .
$$

Therefore by Lemma 3.5, it follows that

$$
\begin{aligned}
S_{3}=\Psi(x, y)\left\{A+O\left(\frac{\log u}{\log y}\right.\right. & +\frac{\log _{2} y}{\log y}+\frac{\log z \log _{2} z}{\log x} \\
& \left.\left.+r e^{-(1-2 r) \log u}+e^{-1 / r}+\frac{e^{r \xi(u)}}{z}\right)\right\} .
\end{aligned}
$$

Now we estimate $S_{4}$. Using Lemmas 2.1 and 3.2 , we can show

$$
\begin{aligned}
S_{4} & \ll \sum_{y<\varphi(n) \leq x / z, P(n) \leq z} \Psi\left(\frac{x}{\varphi(n)}, y\right) \prod_{p \leq z}\left(1-p^{-\alpha_{\varphi(n)}}\right) \\
& \ll \sum_{y<\varphi(n) \leq x / z, P(n) \leq z} \Psi\left(\frac{x}{\varphi(n)}, y\right) \prod_{p \leq z}\left(1-p^{-\beta_{\varphi(n)}}\right) .
\end{aligned}
$$

In addition, Lemma 2.5 and (3.7) allow us to deduce that 


$$
\begin{aligned}
S_{4} & \ll \Psi(x, y) \sum_{y<\varphi(n) \leq x / z, P(n) \leq z} \varphi(n)^{-\beta+c_{1} / \log y} \prod_{p \leq z}\left(1-p^{-\beta_{\varphi(n)}}\right) \\
& \ll \Psi(x, y) \sum_{\varphi(n)>y, P(n) \leq z} \varphi(n)^{-\beta+c_{1} / \log y} \prod_{p \leq z}\left(1-p^{-\beta}\right) .
\end{aligned}
$$

Thus applying Lemma 3.4 leads to the inequality

$$
S_{4} \ll e^{-1 / r} \Psi(x, y) .
$$

It remains to control $A(x, z)$. Since $\varphi(n) \geq c_{6} n / \log _{2} n$, Lemma 2.7 yields

$$
A(x, z) \leq \Psi\left(c_{7} x \log _{2} x, z\right) \ll \Psi\left(x \log _{2} x, z\right) .
$$

Putting $u^{\prime}:=\log \left(x \log _{2} x\right) / \log z$, we have $u^{\prime} \geq u / r \geq 3 u$. Hence Lemma 2.2 implies

$$
\Psi\left(x \log _{2} x, z\right) \ll x\left(\log _{2} x\right) \varrho\left(u^{\prime}\right)^{5 / 6} .
$$

Using the inequalities

$$
u^{-2 u} \ll \varrho(u) \ll u^{-u} \quad(u \geq 1)
$$

which are a simple consequence of (1.5), we may deduce that

$$
\begin{aligned}
\Psi\left(x \log _{2} x, z\right) & \ll x u^{\prime-5 u^{\prime} / 6} \log _{2} x \ll x u^{-5 u^{\prime} / 6} r^{5 u^{\prime} / 6} \log _{2} x \\
& \ll x u^{-5 u / 2} r^{5 u / 2} \log _{2} x \ll x \varrho(u)^{5 / 4} r^{5 u / 2} \log _{2} x .
\end{aligned}
$$

Thus Lemma 2.2 yields that

$$
\Psi\left(x \log _{2} x, z\right) \ll \Psi(x, y) r^{5 u / 2} \log _{2} x \ll \Psi(x, y) r^{5} \log _{2} x .
$$

Combining (3.12) and (3.13) with (3.15), we obtain the desired result.

We can now complete the proof of Theorem 1.

When $y$ is bounded, the result follows by trivial arguments. Hence we may suppose $y \geq y_{0}$, where $y_{0}:=y_{0}(\varepsilon)$ is a sufficiently large constant depending on $\varepsilon$.

First we consider the case

$$
y \geq y_{0}, \quad 2 \leq u \leq \exp \left\{\varepsilon^{-1} \sqrt{\log y}\right\} .
$$

Let $z$ be a parameter to be chosen later which satisfies

$$
(u \log y)^{1+\varepsilon} \leq z \leq \min \left\{\exp \left\{(\log y)^{1 / 2+\varepsilon / 5}\right\}, \exp \left\{\varepsilon^{-3} \log y / \xi(u)\right\}\right\} .
$$

The relation (1.6) and Lemmas 3.6 and 2.7 allow us to write $A(x, y)=$ $\Psi(x, y)\left\{A+O_{\varepsilon}\left(R_{2}\right)\right\}$, where

$$
\begin{aligned}
R_{2}:= & \frac{\log u}{\log y}+\frac{\log _{2} y}{\log y}+\frac{\log z \log _{2} z}{\log x}+r e^{-(1-2 r) \log u} \\
& +e^{-1 / r}+\frac{e^{r \xi(u)}}{z}+r^{5} \log _{2} x+\frac{\log x}{z} .
\end{aligned}
$$


If $2 \leq u \leq \log y$, for the choice $z=(u \log y)^{2}$ we obtain

$$
A(x, y)=\Psi(x, y)\left\{A+O_{\varepsilon}\left(\frac{\log _{2} y \log _{3} y}{\log y}\right)\right\} .
$$

When $\log y<u \leq(\log y)^{1 / \varepsilon}$, we take $z=u^{3}$. Noticing that $\xi(u) \sim \log u$ for $u \rightarrow \infty$ (cf. Lemma 2.2 of [9]), it is easy to verify that this value of $z$ satisfies (3.16). We have

$$
A(x, y)=\Psi(x, y)\left\{A+O_{\varepsilon}\left(\frac{\log u}{\log y}\right)\right\} .
$$

If $(\log y)^{1 / \varepsilon}<u \leq \exp \left\{\varepsilon^{-1} \sqrt{\log y}\right\}$, the choice $z:=\exp \left\{\left(2 \varepsilon^{-1}+1\right) \sqrt{\log y}\right\}$ gives the required result.

Next we consider the case

$$
y \geq y_{0}, \quad 1 \leq u<2 .
$$

We observe that

$$
A(x, y)=\sum_{\varphi(n) \leq x} 1-\sum_{\varphi(n) \leq x, y<P(n) \leq x} 1=A(x)-\sum_{y<p \leq x} A(x /(p-1)) .
$$

Using (1.1) and (1.2), we easily show that

$$
A(x, y)=A x(1-\log u)+O\left(x / \log y+x R_{3}\right),
$$

where

$$
R_{3}:=\sum_{y<p \leq x} \frac{1}{p} \exp \{-\sqrt{\log (x / p)}\}
$$

On the one hand, the prime number theorem allows us to deduce that

$$
R_{3} \ll \int_{y}^{x} \frac{e^{-\sqrt{\log (x / t)}}}{t \log t} d t .
$$

On the other hand, the change of variables $v:=\log x-\log t$ yields that

$$
R_{3} \ll \frac{1}{\log x} \int_{0}^{(1-1 / u) \log x} \frac{e^{-\sqrt{v}}}{1-v / \log x} d v \ll \frac{1}{\log x} \int_{0}^{\infty} e^{-\sqrt{v}} d v \ll \frac{1}{\log x} .
$$

This proves that

$$
A(x, y)=x(1-\log u)\left\{A+O\left(\frac{1}{\log x}\right)\right\} .
$$

By noticing that $\varrho(u)=1-\log u(1 \leq u \leq 2)$, Lemma 2.3 implies

$$
A(x, y)=x \varrho(u)\left\{A+O\left(\frac{1}{\log x}\right)\right\}=\Psi(x, y)\left\{A+O\left(\frac{1}{\log y}\right)\right\} .
$$

This completes the proof of Theorem 1. 
4. Proof of Theorem 2. In view of (1.8), it is sufficient to prove

$$
\sum_{\varphi(n) \leq x} \frac{1}{P(n)}=\left\{A+O\left(\left(\frac{\log _{2} x}{\log x}\right)^{1 / 2}\right)\right\} \sum_{n \leq x} \frac{1}{P(n)} .
$$

Put $I:=\left[L_{-}, L_{+}\right]$, where $L_{-}, L_{+}$are defined as in Lemma 2.8. We split the range of summation into two parts: $\varphi(n) \leq x, P(n) \in I$, and $\varphi(n) \leq$ $x, P(n) \notin I$. The corresponding contributions are denoted by $T_{1}$ and $T_{2}$, respectively.

We first estimate $T_{2}$. For $\varphi(n) \leq x$ and $P(n) \notin I$, we write $n=P(n)^{\nu} m$ with $P(m)<P(n)$. Since $\varphi(n) \leq x$ implies $n \leq c_{8} x \log _{2} x$, we have $\nu \leq$ $(\log n) / \log P(n) \leq c_{9}(\log x) / \log P(n)$. Thus it follows that

$$
\begin{aligned}
T_{2} & =\sum_{p \notin I} \sum_{1 \leq \nu \leq c_{9}(\log x) / \log p} \frac{1}{p} \sum_{\varphi(m) \leq x / p^{\nu-1}(p-1), P(m)<p} 1 \\
& \ll \log x \sum_{p \notin I} \frac{1}{p \log p} A\left(\frac{x}{p-1}, p\right) .
\end{aligned}
$$

In order to estimate the last sum, we decompose it into four parts:

$$
\begin{aligned}
T_{2,1} & :=\sum_{p \leq M} \frac{1}{p \log p} A\left(\frac{x}{p-1}, p\right), \\
T_{2,2} & :=\sum_{M<p<L_{-}} \frac{1}{p \log p} A\left(\frac{x}{p-1}, p\right), \\
T_{2,3} & :=\sum_{L_{+}<p \leq \sqrt{x}+1} \frac{1}{p \log p} A\left(\frac{x}{p-1}, p\right), \\
T_{2,4} & :=\sum_{\sqrt{x}+1<p \leq x+1} \frac{1}{p \log p} A\left(\frac{x}{p-1}, p\right),
\end{aligned}
$$

where $M:=\exp \{\sqrt{\log x}\}$.

For $T_{2,1}$, by Theorem 1 we have

$$
T_{2,1} \leq \sum_{p \leq M} \frac{1}{p \log p} A(x, M) \ll A(x, M) \ll \Psi(x, M) .
$$

Hence Lemma 2.2, (3.14) and (1.9) imply that

$$
\begin{aligned}
T_{2,1} & \ll x \varrho(\sqrt{\log x})^{4 / 5} \ll x e^{-(2 / 5) \sqrt{\log x} \log _{2} x} \\
& \ll \frac{x \delta(x)}{(\log x)^{2}} \ll \frac{1}{(\log x)^{2}} \sum_{n \leq x} \frac{1}{P(n)} .
\end{aligned}
$$

The sums $T_{2,2}$ and $T_{2,3}$ can be estimated as follows: Applying Theorem 1, 
Lemmas 2.6 and 2.8 , we see that

$$
\begin{aligned}
T_{2,2} & \ll \sum_{M<p<L_{-}} \frac{1}{p} \Psi\left(\frac{x}{p-1}, p\right) \ll \sum_{M<p<L_{-}} \frac{1}{p} \Psi\left(\frac{x}{p}, p\right) \\
& \ll \frac{1}{(\log x)^{2}} \sum_{n \leq x} \frac{1}{P(n)}
\end{aligned}
$$

and

$$
\begin{aligned}
T_{2,3} & \ll \sum_{L_{+}<p \leq \sqrt{x}+1} \frac{1}{p} \Psi\left(\frac{x}{p-1}, p\right) \ll \sum_{L_{+}<p \leq \sqrt{x}+1} \frac{1}{p} \Psi\left(\frac{x}{p}, p\right) \\
& \ll \frac{1}{(\log x)^{2}} \sum_{n \leq x} \frac{1}{P(n)} .
\end{aligned}
$$

It is very easy to estimate $T_{2,4}$ :

$$
\begin{aligned}
T_{2,4} & \ll x \sum_{\sqrt{x}+1<p \leq x+1} \frac{1}{(p-1) p \log p} \ll \frac{\sqrt{x}}{(\log x)^{2}} \\
& \ll \frac{1}{(\log x)^{2}} \sum_{n \leq x} \frac{1}{P(n)} .
\end{aligned}
$$

Combining (4.2)-(4.5) leads to the inequality

$$
T_{2} \ll \frac{1}{\log x} \sum_{n \leq x} \frac{1}{P(n)} .
$$

Next we evaluate $T_{1}$. For this, we write

$$
\begin{aligned}
T_{1} & =\sum_{p \in I} \frac{1}{p} \sum_{\substack{\varphi(n) \leq x \\
P(n)=p, p \| n}} 1+\sum_{p \in I} \frac{1}{p} \sum_{\substack{\varphi(n) \leq x \\
P(n)=p, p^{2} \mid n}} 1 \\
& =\sum_{p \in I} \frac{1}{p} \sum_{\substack{\varphi(m) \leq x /(p-1) \\
P(m) \leq p}} 1-\sum_{p \in I} \frac{1}{p} \sum_{\substack{\varphi(m) \leq x /(p-1) \\
P(m)=p}} 1+\sum_{p \in I} \frac{1}{p} \sum_{\substack{\varphi(n) \leq x \\
P(n)=p, p^{2} \mid n}} 1 .
\end{aligned}
$$

Denote by $T_{1,1}, T_{1,2}, T_{1,3}$ the three sums on the right-hand side of (4.7), respectively. For $p \in I$, it is easy to verify that $x /(p-1), p$ satisfy the condition (1.4). Thus Theorem 1 and Lemma 2.7 yield immediately that

$$
\begin{aligned}
T_{1,1} & =\sum_{p \in I} \frac{1}{p} A\left(\frac{x}{p-1}, p\right)=\left\{A+O\left(\left(\frac{\log _{2} x}{\log x}\right)^{1 / 2}\right)\right\} \sum_{p \in I} \frac{1}{p} \Psi\left(\frac{x}{p-1}, p\right) \\
& =\left\{A+O\left(\left(\frac{\log _{2} x}{\log x}\right)^{1 / 2}+\frac{1}{L_{-}}\right)\right\} \sum_{p \in I} \frac{1}{p} \Psi\left(\frac{x}{p}, p\right) .
\end{aligned}
$$


Therefore Lemma 2.8 implies

$$
T_{1,1}=\left\{A+O\left(\left(\frac{\log _{2} x}{\log x}\right)^{1 / 2}\right)\right\} \sum_{n \leq x} \frac{1}{P(n)} .
$$

By Theorem 1 and Lemma 2.6, we have

$$
\begin{aligned}
T_{1,2} & \ll \log x \sum_{p \in I} \frac{1}{p} A\left(\frac{x}{(p-1)^{2}}, p\right) \\
& \ll \log x \sum_{p \in I} \frac{1}{p} \Psi\left(\frac{x}{(p-1)^{2}}, p\right) \ll \log x \sum_{p \in I} \frac{1}{p} \Psi\left(\frac{x}{p^{2}}, p\right)
\end{aligned}
$$

and

$$
\begin{aligned}
T_{1,3} & \ll \log x \sum_{p \in I} \frac{1}{p} A\left(\frac{x}{p(p-1)}, p\right) \\
& \ll \log x \sum_{p \in I} \frac{1}{p} \Psi\left(\frac{x}{p(p-1)}, p\right) \ll \log x \sum_{p \in I} \frac{1}{p} \Psi\left(\frac{x}{p^{2}}, p\right) .
\end{aligned}
$$

For $p \in I$, we easily see that $\beta(x / p, p)=1+O\left(\log _{2} x / \sqrt{\log x}\right)>1 / 2$. Thus Lemmas 2.4 and 2.8 yield that

$$
\sum_{p \in I} \frac{1}{p} \Psi\left(\frac{x}{p^{2}}, p\right) \ll \frac{1}{\sqrt{L_{-}}} \sum_{p \in I} \frac{1}{p} \Psi\left(\frac{x}{p}, p\right) \ll \frac{1}{\sqrt{L_{-}}} \sum_{n \leq x} \frac{1}{P(n)} .
$$

Inserting these estimates in (4.7), we obtain

$$
T_{1}=\left\{A+O\left(\left(\frac{\log _{2} x}{\log x}\right)^{1 / 2}\right)\right\} \sum_{n \leq x} \frac{1}{P(n)} .
$$

Now the desired result (4.1) follows from (4.6) and (4.8).

5. Proof of Theorem 3. Let $U$ be the sum to be evaluated. We have

$$
U=A(x) \log x-\sum_{\varphi(n) \leq x} \int_{P(n)}^{x} \frac{d y}{y}=A(x) \log x-\int_{1}^{x} A(x, y) \frac{d y}{y} .
$$

To estimate the last integral, we decompose it into two parts:

$$
U_{1}:=\int_{1}^{\exp \left\{\left(\varepsilon \log _{2} x\right)^{2}\right\}} A(x, y) \frac{d y}{y}, \quad U_{2}:=\int_{\exp \left\{\left(\varepsilon \log _{2} x\right)^{2}\right\}}^{x} A(x, y) \frac{d y}{y} .
$$

Using the trivial inequality (see Theorem III.5.1 of [14])

$$
A(x, y) \ll \Psi\left(x \log _{2} x, y\right) \ll x\left(\log _{2} x\right) e^{-u / 2},
$$


we can find that

$$
U_{1} \ll_{\varepsilon} x \exp \left\{-(\log x)^{1-\varepsilon}\right\} .
$$

For $U_{2}$, Corollary 2 allows us to write $U_{2}=A x U_{2}^{\prime}+O\left(x U_{2}^{\prime \prime}\right)$, where

$$
\begin{aligned}
U_{2}^{\prime} & :=\int_{\exp \left\{\left(\varepsilon \log _{2} x\right)^{2}\right\}}^{x} \varrho\left(\frac{\log x}{\log y}\right) \frac{d y}{y}, \\
U_{2}^{\prime \prime} & :=\int_{\exp \left\{\left(\varepsilon \log _{2} x\right)^{2}\right\}}^{x} \varrho\left(\frac{\log x}{\log y}\right) \frac{\log (\log x / \log y)+\log _{2} y \log _{3} y}{\log y} \frac{d y}{y} .
\end{aligned}
$$

By the change of variables $t:=\log x / \log y$, a simple calculation shows that

$$
\begin{aligned}
& U_{2}^{\prime}=\int_{1}^{\infty} \varrho(t) t^{-2} d t \log x+O_{\varepsilon}\left(\exp \left\{-(\log x)^{1-\varepsilon}\right\}\right), \\
& U_{2}^{\prime \prime} \ll \int_{1}^{(\log x) /\left(\varepsilon \log _{2} x\right)^{2}} \varrho(t) \frac{\log t+\log _{2} x \log _{3} x}{t} d t \ll \log _{2} x \log _{3} x,
\end{aligned}
$$

which implies

$$
U_{2}=A \int_{1}^{\infty} \varrho(t) t^{-2} d t x \log x+O\left(x \log _{2} x \log _{3} x\right) .
$$

Inserting (5.2)-(5.3) in (5.1) and using (1.2), we obtain the result of Theorem 3.

\section{References}

[1] M. Balazard and A. Smati, Elementary proof of a theorem of Bateman, in: Analytic Number Theory, Proceedings of a Conference in Honor of Paul T. Bateman, B. Berndt, H. Diamond, H. Halberstam and A. Hildebrand (eds.), Urbana, 1989, Progr. Math. 85, Birkhäuser, 1990, 41-46.

[2] P. T. Bateman, The distribution of values of Euler's function, Acta Arith. 21 (1972), 329-345.

[3] N. G. deBruijn, The asymptotic behaviour of a function occurring in the theory of primes, J. Indian Math. Soc. (N.S.) 15 (1951), 25-32.

[4] - On the number of positive integers $\leq x$ and free of prime factors $>y, I I$, Nederl. Akad. Wetensch. Proc. Ser. A 69 (1966), 239-247.

[5] P. Erdős, Some remarks on Euler's $\phi$-function and some related problems, Bull. Amer. Math. Soc. 51 (1945), 540-544.

[6] P. Erdős, A. Ivić and C. Pomer ance, On sums involving reciprocals of the largest prime factor of an integer, Glas. Mat. Ser. III 21 (41) (1986), 283-300.

[7] A. Hildebrand, On the number of positive integers $\leq x$ and free of prime factors $>y$, J. Number Theory 22 (1986), 289-307.

[8] A. Hildebrand and G. Tenenbaum, On integers free of large prime factors, Trans. Amer. Math. Soc. 296 (1986), 265-290. 
[9] A. Hildebrand and G. Tenenbaum, Integers without large prime factors, J. Théor. Nombres Bordeaux 5 (1993), 411-484.

[10] A. Ivić, On some estimates involving the number of prime divisors of an integer, Acta Arith. 49 (1987), 21-33.

[11] A. Ivić and G. Tenenbaum, Local densities over integers free of large prime factors, Quart. J. Math. Oxford Ser. (2) 37 (1986), 401-417.

[12] E. Saias, Entiers sans grand ni petit facteur premier III, Acta Arith. 71 (1995), 351-379.

[13] A. Smati and J. W u, Distribution of values of Euler's function in algebraic number fields, Prépublication du Laboratoire d'Arithmétique, Calcul Formel \& Optimisation, Université Limoges, 94/ $\mathrm{N}^{\circ} 4$.

[14] G. Tenenbaum, Introduction to Analytic and Probabilistic Number Theory, Cambridge University Press, 1995.

Département de Mathématiques Unité associée au CNRS, URA 1586

Université Limoges

87060 Limoges Cedex, France

E-mail: smati@cict.fr
Laboratoire de Mathématiques Institut Élie Cartan - UMR CNRS 9973 Université Henri Poincaré, Nancy 1 54506 Vandœuvre-lès-Nancy, France E-mail: wujie@iecn.u-nancy.fr 